a minería es una de las actividades

económicas de mayor tradición en México, la cual ha contribuido desde la época prehispánica hasta nuestros tiempos al desarrollo del país (Contreras-Díaz et al., 2000; González, et al., 2005; Hernández-Acosta et al., 2009). Esta producción minera en México, se concentra en trece entidades: Chihuahua, Jalisco, Michoacán, Zacatecas, Durango, Sonora, Coahuila, Guanajuato, San Luis Potosí, Hidalgo, Sinaloa, Colima y Guerrero (CarrilloGonzález, 2005). La Unidad Minera de Taxco (UMT) se ha caracterizado históricamente por su producción de plata. Sin embargo, a partir de las dos primeras décadas del siglo XX con la introducción del proceso de flotación, se convierte en un importante productor de $\mathrm{Zn}$ y $\mathrm{Pb}$ (Romero-Martin, Com. Pers.). Pero esta industria minera ha generado por décadas una gran cantidad de desechos mineros o jales, los cuales pueden ser definidos como lodos residuales originados durante el proceso de beneficio del mineral económicamente aprovechable (González et al., 2005). Los jales "La Concha" y "El Fraile" son localizados aproximadamente a $12 \mathrm{Km}$ del Suroeste de la ciudad de Taxco y contienen desechos mineros originados entre 1940 y 1970 (Talavera, 2006).

Uno de los costos ambientales de la actividad minero metalúrgica es la contaminación del medio abiótico (agua, suelo, subsuelo, aire, sedimentos). Son escasos los estudios sobre las especies vegetales que pueden crecer en suelos contaminados por residuos de minas (Olguin et al., 2003; Flores-Tavison et al., 2003; Carrillo-González, 2005; Armienta et al., 2008; Hernández-Acosta et al., 2009). También es mínima la información sobre las concentraciones de elementos que pueden acumular las especies (Carrillo-González, 2005; Prieto-García et al., 2005; Armienta et al., 2008; Hernández-Acosta et al., 2009) así como sus mecanismos de adaptación (González, 2005), y si los

\section{Plantas de Sitios CONTAMINADOS CON DESECHOS MINEROS EN Taxco, Guerrero, México}

\author{
Juan Miguel Gómez-Bernal ${ }^{1,4}$, 5 , Jorge Santana-Carillo², \\ Fracisco Romero-Martin³, María Aurora Armienta-Hernández ${ }^{4}$, \\ Ofelia Morton-Bermea ${ }^{4}$ y Esther Aurora Ruiz-Huerta ${ }^{1}$ \\ ${ }^{1}$ Posgrado en Ciencias de la Tierra, Universidad Nacional Autónoma de México \\ ${ }^{2}$ Herbario Metropolitano, Departamento de Biología, \\ Universidad Autónoma de México \\ ${ }^{3}$ Instituto de Geología, Universidad Nacional Autónoma de México \\ ${ }^{4}$ Instituto de Geofísica, Universidad Nacional Autónoma de México \\ ${ }^{5}$ Autor para la correspondencia: gupi02@yahoo.com.mx
}

acumulan en sus estructuras o evitan que los contaminantes penetren la raíz. Flores-Tavison et al. (2003) reportaron algunas especies que crecen en suelos contaminados por arsénico. Bailleres (2003) reportó Pluchea symphytifolia (Mill.) Gillis creciendo exitosamente en un matorral sobre residuos de minas. Díaz-Garduño et al. (2005) recolectó 44 especies de vegetales en cuatro jales de Zacatecas y 29 especies vegetales en tres sitios de Temascaltepec, Estado de México. Carrillo y González-Chávez (2005) reportaron a Polygonum aviculare L. y Jatropha dioica Sessé como acumuladoras de zinc. Armienta et al. (2008) registró Prosopis laevigata L. y Acacia farnesiana (L.) Willd. en suelos ricos con arsénico en Zimapán, Hidalgo. Hernández-Acosta et al., (2009) identificaron 25 especies de plantas, donde Haplopappus venetus (Kunth) S.F. Blake fue la más dominante en un jal de mina en Pachuca, Hidalgo. Así mismo, se han detectado varias zonas mineras contaminadas en el país que pueden causar problemas de salud hu-

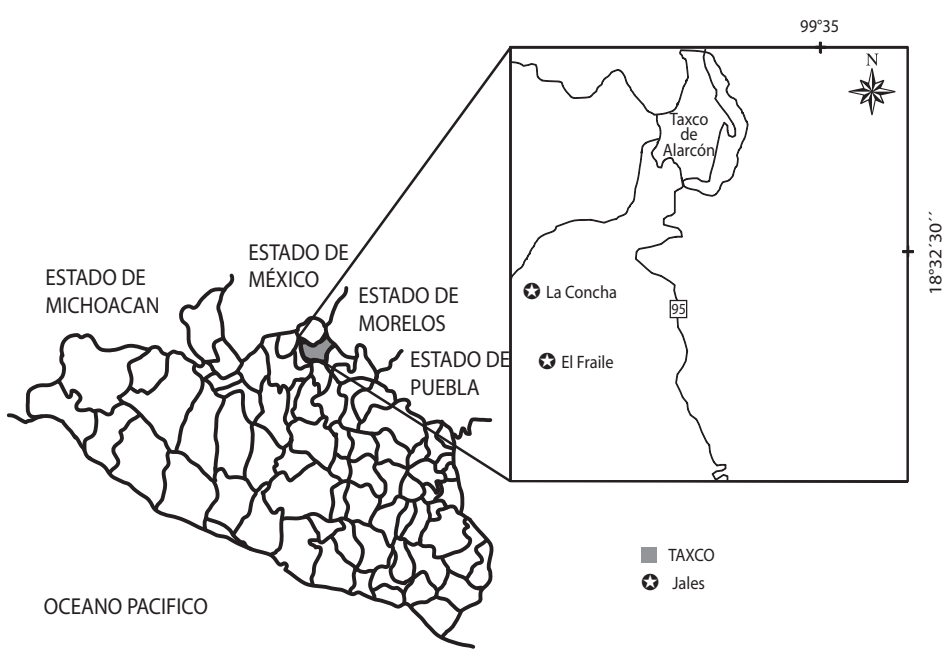

Figura 1. Localización del municipio de Taxco de Alarcón, Guerrero. 
Cuadro 1. Especies recolectadas en los jales "La Concha" (LC) y "El Fraile" (EF) en el municipio de Taxco de Alarcón, Guerrero. P (Planicie del jal), B (Base del jal), Hm (Humedal). Forma de vida: H (Hierba), A (Árbol), Ar (Arbusto), E (Epífita), F (Fanerófita), Hi (Hidrófita).

1. Acacia cochliacantha Humb. \& Bonpl. ex Willd. (Leguminosae) A LC B

2. Annona squamosa L. (Annonaceae) A EF B

3. Bouteloua repens (Kunth) Scribn. \& Merr. (Poaceae) H LC P, Hm

4. Buddleja cordata Kunth (Loganiaceae) Ar EF B

5. Cupressus lindleyi Klotzsch ex Endl. (Cupressaceae) A LC P,B EF P, B

6. Dodonaea viscosa Jacq. (Sapindaceae) H LC B

7. Gibasis pulchella (Kunth) Raf. (Commelinaceae) H LC B

8. Gnaphalium canescens DC. (Asteraceae) H LC P

9. Gomphena decumbens Jacq. (Amaranthaceae) H LC $\mathbf{P}$

10. Hypericum philonotis Schltdl. \& Cham. (Hypericaceae) H LC B

11. Ipomoea nil (L.) Roth (Convolvulaceae) H LC B EF P

12. Iresine celosia L. (Amaranthaceae) H LC B

13. Jacaranda mimosifolia D. Don (Bignoniaceae) A LC $\quad \mathbf{P}, \mathbf{B} \quad$ EF $\quad \mathbf{P}$

14. Juniperus deppeana Steud. (Cupressaceae) Ar LC P,B EF P,B

15. Juniperus flaccida Schltdl. (Cupressaceae) Ar LC P EF $\mathbf{P}$

16. Leucena aff. diversifolia (Scld.) Benth. (Leguminosae) A LC B

17. Lysiloma acapulcense (Kunth) Benth. (Leguminosae) Ar EF B

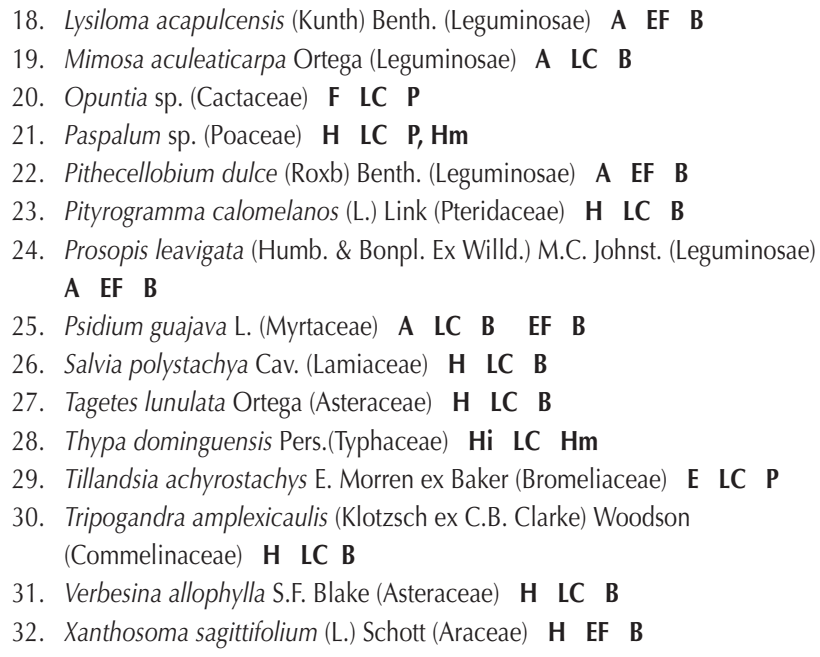

mana y ecotoxicológicos a mediano y largo plazo, como los observados en Gómez Palacio, Durango, en la presa "La Zacatecana" en Zacatecas, o en San Luis Potosí.

Algunos casos de restauración de sitios contaminados incluyen el recubrimiento de los residuos para reducir la dispersión de contaminantes. Por ejemplo en Zimapán, Hidalgo se cubrieron con suelo dos presas de jales drenados y secos, y se sembraron algunas especies de árboles (eucaliptos y casuarinas) y pastos, para reducir la dispersión de los residuos contaminantes por erosión. A su vez, también se realizaron trabajos similares en Taxco, Guerrero, donde se sembró Prosopis leavigata L. en los jales "El Fraile" para amortiguar la dispersión de los contaminantes (Obs. Pers.). El objetivo de este trabajo es identificar las especies vegetales que colonizan las áreas perturbadas por la minería en dos jales de Taxco, Guerrero. Los resultados de este inventario se podrán utilizar para medidas de fitoremediación.

Taxco de Alarcón se localiza al norte de la capital en el estado de Guerrero a 1,752 m s.n.m. (Figura 1). El clima de la zona es de tipo ACw2" (w)ig, semicálido, subhúmedo con lluvias en verano, con canícula y poca oscilación térmica (García, 2004). La vegetación que prevalece es el bosque tropical caducifolio (Martínez, et al., 2004; Hinojosa-Espinosa y Cruz-Durán, 2010).

Se recolectaron ejemplares botánicos durante el 2008, utilizando el método de cuadrados. Se estudiaron dos áreas de influencia minera: el jal "La Concha" y el jal "El Fraile". El material recolectado se incorporó al Herbario Metropolitano UAMIZ.

En total se colectaron plantas correspondientes a 31 especies. Las familias mejor representadas de plantas son Fabaceae y Cupressaceae. Las plantas colectadas en el jal "La Concha" pertenecen a 23 especies de 16 familias (Cuadro 1). Las mejor representadas fueron: Asteraceae, Cupressaceae, Amaranthaceae, Commelinaceae y Fabaceae. Las colectadas en el jal "El Fraile", corresponden a 15 especies (Cuadro 1). La vegetación circundante consta de seis tipos de comunidades vegetales, de los cuales el bosque tropical caducifolio es el más abundante, seguido del bosque de coníferas, el bosque mesófilo de montaña, el bosque de galería y finalmente los pastizales con humedales semipermanentes.

Los desechos mineros favorecen la presencia de ciertas plantas de asociaciones vegetales secundarias (e.g.
Bouteloua repens Kunth, Gomphena decumbens Jacq) y también especies del bosque primario, como por ejemplo las especies pioneras Juniperus deppeana Steud, Juniperus flaccida Schltdl y Cupressus lindleyi Klotzsch ex Endl. De modo que para lograr una restauración adecuada es conveniente conocer los procesos de sucesión vegetal. Sugerimos que en la restauración de estos sitios mineros en la zona de Taxco, se consideren las especies que encontramos en este estudio.

\section{Agradecimientos}

Los autores reconocen la participación de Mario Adolfo Espejo Serna de la Universidad Autónoma Metropolitana Unidad Iztapalapa por sus atinados comentarios para mejorar el presente artículo, así como a Fernando Salgado Mejía estudiante de servicio social por su participación en la recolección de especies vegetales en las zonas de estudio.

\section{Literatura citada}

Armienta M.A., Ongley L.K., Rodríguez R., Cruz O., Mango H. y Villaseñor G. 2008. Arsenic distribution in mesquite (Prosopis laevigata) and huizache (Acacia farnesiana) in the Zimapán mining area, México. Geochemistry: Explora- 
tion, Environment, Analysis 8:1-7.

Bailleres A. 2003. Hacia un desarrollo sustentable. Informe anual ambiental 2002. Industrias Peñoles S.A. de C.V. [En línea]. <http:/www.penoles.com. $\mathrm{mx}>$ (Consultado noviembre 2003).

Carrillo-González R. 2005. Niveles de contaminación de los suelos y las plantas. En: González-Chávez M.C. PérezMoreno J. y Carrillo-González R. Eds. El sistema planta-microorganismo-suelo en áreas contaminadas con residuos de minas, pp. 161 Colegio de Postgraduados. Montecillo, estado de México.

Carrillo-González R. y González-Chávez M.C.A. 2006. Metal accumulation in wild plants surrounding mining wastes. Environmental Pollution 144:84-92.

Contreras Díaz F.J., Ortiz-Romero J.R. y Padilla M. 2000. Unidad Académica de Economía de la Universidad Autónoma de Zacatecas.<http://www.ciu.reduaz. mx/Pruebas.nsf/ b4c56a1adbc9e5f8625 6ac300614816/ 8ad4c2450a00aeab862 56ec8a2?OpenDocument $>$ (Consultado 24 mayo 2004).

Díaz-Garduño M., Díaz-Osornio A., Carrillo-González R. y González-Chávez M.C. 2005. Plantas que se desarrollan en áreas contaminadas con residuos mineros. En: González-Chávez M.C., Pérez-Moreno J. y Carrillo-González
R. Eds. El Sistema Planta-Microorganismo-Suelo en Áreas Contaminadas con Residuos de Minas, pp. 9-33, Colegio de Postgraduados. Montecillo, México.

Flores-Tavison E., Alarcon-Herrera M.T., González E.S. y Olguín E.J. 2003. Arsenic tolerating plants from mine sites and hot springs in the semi Arid region of Chihuahua, México. Acta biotechnol 23:113-119.

García, E. 2004. Modificaciones al sistema de clasificación climática de Koppen para adaptarlo a las condiciones de la República Mexicana. Instituto de Geografía, UNAM. México, D.F.

González-Chávez M.C. 2005. Recuperación de suelos contaminados con metales pesados utilizando plantas y microorganismos rizosféricos. Terra Latinoamerica 23:29-37.

González Chávez M.C., Pérez M.J. y Carillo-González R. 2005. El sistema planta-Microorganismo-suelo en áreas contaminadas con residuos de minas. Colegio de posgraduados. Texcoco, México.

Hernández-Acosta E., Mondragón-Romero E., Cristobal-Acevedo D., RubiñosPanta J.E. y Robledo-Santoyo. 2009. Vegtación, residuos de mina y elementos potencialmente tóxicos de un jal de Pachuca, Hidalgo, México. Revista
Chapingo Serie Forestal y del Ambiente 15:109-14.

Hinojosa-Espinosa O. y Cruz-Durán R. 2010. Compositae en Atenango del Río, Guerrero, México. Boletín de la Sociedad Botánica de México 86:71-73.

Martínez G.M., Cruz D.R., Castrejón R.J.F., Valencia A.S., Jiménez R.J. y Ruiz-Jiménez C.A. 2004. Flora vascular de la porción guerrerense de la sierra de Taxco, Guerrero, México. Anales del Instituto de Biología. Serie Botánica, 75:105-189.

Prieto-García F., Callejas H.J., Lechuga M.A., Gaytán J.C. y Barrado E.E. 2005. Acumulación en tejidos vegetales de Arsénico provenientes de aguas y suelos de Zimapán estado de Hidalgo, México. Bioagro 17:129-135.

Talavera M.O., Armienta M.A., García A.J. y Flores M.N. Geochemistry of leachates from the El Fraile sulfide tailings piles in Taxco, Guerrero, southern Mexico. Environmental Geochemistry and Health 28:243-255.

Recibido: 15 de junio de 2010

Aceptado: 2 de noviembre de 2010 